\title{
Connections between the Generalized Marcum $Q$-Function and a class of Hypergeometric Functions
}

\author{
D. Morales-Jimenez, F. J. Lopez-Martinez, E. Martos-Naya, J. F. Paris, and A. Lozano
}

\begin{abstract}
This paper presents a new connection between the generalized Marcum- $Q$ function and the confluent hypergeometric function of two variables, $\Phi_{3}$. This result is then applied to the closed-form characterization of the bivariate Nakagami- $m$ distribution and of the distribution of the minimum eigenvalue of correlated non-central Wishart matrices, both important in communication theory. New expressions for the corresponding cumulative distributions are obtained and a number of communication-theoretic problems involving them are pointed out.
\end{abstract}

\section{Index Terms}

Marcum- $Q$ function, confluent hypergeometric functions, bivariate Nakagami- $m$, non-central Wishart matrix, minimum eigenvalue distribution.

\section{INTRODUCTION}

The number of special functions that make appearances in the communication theory arena continues to grow. Some of these are tabulated and well studied, with readily available approximations, bounds,

This work has been submitted to the IEEE for possible publication. Copyright may be transferred without notice, after which this version may no longer be accessible

D. Morales-Jimenez and A. Lozano are with the Department of Information and Communication Technologies, Universitat Pompeu Fabra, 08018 Barcelona (Spain). (e-mail:\{d.morales,angel.lozano\}@upf.edu)

F. J. Lopez-Martinez is with the Wireless Systems Lab, Stanford University, Stanford, CA 94305-9515 (USA). (e-mail: fjlm@stanford.edu)

E. Martos-Naya and J. F. Paris are with Dept. Ingenieria de Comunicaciones, University of Malaga, 29071 Malaga (Spain). (e-mail: \{eduardo,paris\}@ic.uma.es) 
and asymptotic expansions. Other special functions, however, are not so well studied and their numerical computation is not always as accurate and efficient as would be desirable. In such cases, the establishment of connections with other special functions can greatly reinforce the analytical toolbox available to researchers.

A wealth of works have studied the Marcum- $Q$ function (see, e.g., [1]-[5]), deriving useful bounds and approximations and evidencing applications thereof. Routines for its accurate and efficient evaluation have been extensively developed and, in fact, the generalized Marcum- $Q$ function is included in most common mathematical software packages.

Confluent hypergeometric functions also appear in a fair number of problems within communication theory and signal processing [6]-[9] [10]. Moreover, recent distributional results [8], [11] show that a number of other such problems (see, e.g., [7], [12]-[22]) can be solved in terms of confluent hypergeometric functions. Chief among these stands the $\Phi_{3}$ confluent hypergeometric function [23], [24], which does not lend itself to easy and precise evaluation.

This paper shows that $\Phi_{3}$ can be expressed in terms of the generalized Marcum- $Q$ function. This connection is then applied to the closed-form characterization of two important distributions in communicationtheory: the bivariate Nakagami- $m$ distribution, and the distribution of the minimum eigenvalue of correlated non-central Wishart matrices. New expressions for the corresponding cumulative distribution functions (CDFs) are obtained and some communication-theoretic problems involving them are pointed out. In particular, the connection unveiled herein, in combination with recent results in [11], settles the standing conjecture [15] [14, p. 174] that the bivariate Nakagami- $m$ CDF can be expressed in terms of the generalized Marcum- $Q$ function.

\section{PRELiminaries}

This section introduces the special functions under study and recalls a few properties of interest for the derivations that follow.

Definition 1: The generalized Marcum- $Q$ function is defined as [14], [25], [26]

$$
Q_{m}(a, b)=\int_{b}^{\infty} \frac{x^{m}}{a^{m-1}} \exp \left(-\frac{a^{2}+x^{2}}{2}\right) I_{m-1}(a x) d x
$$

where $a>0$ and $b \geq 0$ are real parameters and $I_{m}(\cdot)$ is the $m$ th order modified Bessel function of the first kind. The order index $m$ is an integer and typically $m \geq 0$, yet (1) holds for negative orders too and a useful relationship between Marcum- $Q$ functions with positive and negative orders has been reported 
in [27], namely

$$
Q_{m}(a, b)=1-Q_{1-m}(b, a)
$$

Definition 2: The $\Phi_{3}$ confluent hypergeometric function of two variables is defined as [23, Eq. 9.261.3]

$$
\Phi_{3}(b, c ; w, z)=\sum_{k=0}^{\infty} \sum_{m=0}^{\infty} \frac{(b)_{k}}{(c)_{k+m}} \frac{w^{k} z^{m}}{k ! m !}
$$

where $b, c, w, z \in \mathbb{R}, c \neq 0,-1,-2, \ldots$, and $(t)_{r}=\frac{\Gamma(t+r)}{\Gamma(t)}$ denotes the Pochhammer symbol with $\Gamma(\cdot)$ the Gamma function. The $\Phi_{3}$ function is one of the bivariate forms of the confluent hypergeometric function ${ }_{1} F_{1}(\cdot, \cdot ; \cdot)$ [23]. Note that $\Phi_{3}$ does not exist for non-positive integer values of $c$ due to the singularities of the Gamma function. Next, we introduce a regularized version of this function, which is valid for any $c \in \mathbb{R}$.

Definition 3: The regularized $\Phi_{3}$ function is defined as

$$
\begin{aligned}
\tilde{\Phi}_{3}(b, c ; w, z) & =\frac{1}{\Gamma(c)} \Phi_{3}(b, c ; w, z) \\
& =\sum_{k=0}^{\infty} \sum_{m=0}^{\infty} \frac{(b)_{k}}{\Gamma(c+k+m)} \frac{w^{k} z^{m}}{k ! m !}
\end{aligned}
$$

with $b, c, w, z \in \mathbb{R}$. For the special cases $w=0$ and/or $z=0, \tilde{\Phi}_{3}$ reduces to

$$
\begin{aligned}
\tilde{\Phi}_{3}(b, c ; 0, z) & =z^{(1-c) / 2} I_{c-1}(2 \sqrt{z}) \\
\tilde{\Phi}_{3}(b, c ; w, 0) & ={ }_{1} \tilde{F}_{1}(b, c ; w) \\
\tilde{\Phi}_{3}(b, c ; 0,0) & =\frac{1}{\Gamma(c)}
\end{aligned}
$$

where ${ }_{1} \tilde{F}_{1}(b, c ; w)={ }_{1} F_{1}(b, c ; w) / \Gamma(c)$ is the regularized confluent hypergeometric function [23].

The Laplace transforms of $\Phi_{3}$ and $\tilde{\Phi}_{3}$ are known and, due to their simple form, will be crucial in the ensuing derivations. Given the function $f(t)=t^{c-1} \tilde{\Phi}_{3}(b, c ; x t, y t)$, its Laplace transform is given by [24, Eq. 4.24.9]

$$
\mathcal{L}\{f(t) ; s\}=s^{-c}\left(1-\frac{x}{s}\right)^{-b} e^{y / s}
$$

\section{MAIN RESUlT}

The main result, presented in this section, rests on two new lemmas that provide, respectively, a new representation for the generalized Marcum- $Q$ function and a recursive relationship for $\tilde{\Phi}_{3}$.

Lemma 1: The generalized Marcum- $Q$ function can be expressed in terms of $\tilde{\Phi}_{3}$ as

$$
Q_{m}(a, b)=\left(\frac{a^{2}}{2}\right)^{1-m} \exp \left(-\frac{a^{2}+b^{2}}{2}\right) \tilde{\Phi}_{3}\left(1,2-m ; \frac{a^{2}}{2}, \frac{a^{2} b^{2}}{4}\right), \quad m \in \mathbb{Z} .
$$


Proof: See Appendix A.

Lemma 2: The regularized $\Phi_{3}$ function can be obtained recursively as

$$
\tilde{\Phi}_{3}(b, c ; w, z)=\left(\frac{z}{w}\right)^{b-1} \sum_{i=0}^{2(b-1)} \frac{1}{z^{i}} \mathcal{A}_{i}(b, c ; z) \tilde{\Phi}_{3}(1, c-i ; w, z)
$$

for any $b \in \mathbb{Z}$ and $b>0$, with $\mathcal{A}_{i}(b, c ; z)$ being the polynomial on $z$ given by

$$
\mathcal{A}_{i}(b, c ; z)=\frac{(-1)^{b-1}}{(b-1) !} \sum_{k=0}^{\lfloor i / 2\rfloor} \frac{(-1)^{k}(b-i+k)_{i-k}(c-i-1+k)_{i-2 k}}{(i-2 k) ! k !} z^{k} .
$$

Proof: See Appendix B.

Leveraging the foregoing lemmas, the main result in this paper can be put forth.

Theorem 1: The $\tilde{\Phi}_{3}$ function is given in terms of the Marcum- $Q$ function as

$$
\tilde{\Phi}_{3}(b, c ; w, z)=\left(\frac{z}{w}\right)^{b-1} \sum_{i=0}^{2(b-1)} \frac{\mathcal{A}_{i}(b, c ; z)}{w^{c-i-1} z^{i}} \exp \left(w+\frac{z}{w}\right) Q_{2-c+i}\left(\sqrt{2 w}, \sqrt{2 \frac{z}{w}}\right)
$$

with $b, c \in \mathbb{Z}, b>0, z \neq 0, w \neq 0$, and $\mathcal{A}_{i}(b, c ; z)$ given by (11).

Proof: The result follows directly from lemmas 1 and 2 after solving for $\tilde{\Phi}_{3}(1, c-i ; w, z)$ in 9 ) and substituting in $(10)$.

A few comments on the above theorem are in order:

- For the special cases $w=0$ and $z=0$, simpler connections are respectively given in (5) and (6) in terms of $I_{m}(\cdot)$ and ${ }_{1} F_{1}(\cdot, \cdot ; \cdot)$. If $w=z=0$, then 77 gives $\tilde{\Phi}_{3}$ in terms of the Gamma function.

- Albeit $\tilde{\Phi}_{3}(b, c ; w, z)$ is defined for any real value of its arguments, Thm. 1 is restricted to integer values of $b$ and $c$; these are precisely the cases of interest in communication theory. Moreover, the validity of 12 can be straightforwardly extended to $c \in \mathbb{R}$ by applying (34) in Appendix A in place of (9).

- Negative values of $w, z$ (which do not correspond to known communication theory problems) imply complex arguments of the Marcum- $Q$ function. This is not an issue since the Marcum- $Q$ definition in (1) also holds for complex arguments by analytic continuation [1].

Thm. 1 allows expressing any result involving $\Phi_{3}$ in terms of the generalized Marcum- $Q$ function. Besides having archival value, this relationship greatly facilitates both the evaluation of such results, and any subsequent analysis thereof.

\section{ApplicAtions}

The generalized Marcum- $Q$ and the $\Phi_{3}$ functions appear in a number of communication theory problems. The new connection between these functions presented in Thm. 1 can be therefore applied 
directly to such problems. For instance, $\Phi_{3}$ appears in two important distributions: the bivariate Nakagami$m$ distribution, and the distribution of the minimum eigenvalue of non-central Wishart matrices. We next exemplify the applicability of Thm. 1 to these specific problems.

\section{A. Bivariate Nakagami-m Distribution}

Thm. 11, together with recent results for the bivariate Nakagami- $m$ distribution in [11], confirm the conjecture made in [15] and in [14, p. 174]: the joint CDF of two correlated Nakagami- $m$ variables can be expressed in terms of the generalized Marcum- $Q$ function, thereby completing the landscape of (closed-form) bivariate characterizations of the most common fading distributions.

Corollary 1: Let $R_{1}$ and $R_{2}$ be two correlated Nakagami- $m$ random variables with positive integer fading index $m$, respective variances $\Omega_{1}=\mathrm{E}\left[R_{1}^{2}\right]$ and $\Omega_{2}=\mathrm{E}\left[R_{2}^{2}\right]$, and correlation coefficient $\rho$, and let $\hat{R}_{1}$ and $\hat{R}_{2}$ be the normalized (unit-variance) versions of $R_{1}$ and $R_{2}$. Then, the joint CDF of $\hat{R}_{1}$ and $\hat{R}_{2}$ is given by

$$
\begin{aligned}
F_{\hat{R}_{1}, \hat{R}_{2}}\left(r_{1}, r_{2}\right) & =\frac{\gamma\left(m, m r_{2}^{2}\right)}{(m-1) !}-e^{-m r_{1}^{2}} \sum_{k=0}^{m-1} \frac{\left(m r_{1}^{2}\right)^{k}}{k !} Q_{1-k}\left(r_{2} a, r_{1} b\right) \\
& +e^{-m r_{2}^{2}} \sum_{k=0}^{m-1} \sum_{i=1}^{m-k} \sum_{r=0}^{2(i-1)} \frac{(1-\rho)^{r}}{k ! \rho^{r}}\left(\frac{m r_{1}^{2}}{\rho}\right)^{k+i-r-1} \mathcal{A}_{r}\left(i, k+i ; \frac{\left(r_{1} r_{2} a b\right)^{2}}{4}\right) Q_{2-k-i+r}\left(r_{2} b, r_{1} a\right)
\end{aligned}
$$

where $a=\sqrt{\frac{2 m}{1-\rho}}, b=a \sqrt{\rho}, \gamma(\cdot, \cdot)$ is the lower incomplete gamma function [23], and $\mathcal{A}_{r}(\cdot, \cdot ; \cdot)$ are the polynomials defined in 11 . It follows that

$$
F_{R_{1}, R_{2}}\left(r_{1}, r_{2}\right)=F_{\hat{R}_{1}, \hat{R}_{2}}\left(\frac{r_{1}}{\sqrt{\Omega_{1}}}, \frac{r_{2}}{\sqrt{\Omega_{2}}}\right) .
$$

Proof: The bivariate Nakagami- $m$ CDF is given in [11, Eq. 14] in terms of the $\Phi_{3}$ function. Then, (13) is obtained by virtue of Thm. 1 after normalization of the random variables and some algebraic manipulations.

Corollary 2: The well-known expression for the bivariate Rayleigh CDF can be recovered from Corollary 1 by setting $m=1$ and using [23, Eq. 8.352.1] to expand the incomplete Gamma function, yielding

$$
F_{\hat{R}_{1}, \hat{R}_{2}}\left(r_{1}, r_{2}\right)=1-e^{-r_{2}^{2}}-e^{-r_{1}^{2}} Q_{1}\left(r_{2} a, r_{1} b\right)+e^{-r_{2}^{2}} Q_{1}\left(r_{2} b, r_{1} a\right)
$$

consistently with [28, Appendix A].

The closed-form characterization of the bivariate Nakagami- $m$ distribution had remained an open problem for decades. Existing expressions involved infinite summations [15]-[17] or an integral of the 
product of Marcum- $Q$ functions [18]. The expression that recently appeared in [11], in terms of $\Phi_{3}$, has been rewritten by virtue of Thm. 1 into the convenient form given in Corollary 1 . The problems whose analysis can benefit from this form include [11], [14]-[22]:

- Determining the impact of fading correlation in dual-diversity reception or transmission [11], [19] [20, sect. 5.2.5].

- Analyzing the level crossing rate and average fade duration of sampled fading envelopes [11].

- Establishing the transition probabilities for a first-order Markov chain that models a fading process [21], [22]. This, in turn, can be applied to approximate the envelope of channels with non-independent fading [22] or to model the decoding success/failure with automatic repeat-request (ARQ) over successive channel realizations [21].

\section{B. Minimum Eigenvalue Distribution of Non-Central Wishart Matrices}

Definition 4 (Non-central Wishart matrix): Let $\mathbf{X}$ be an $n \times m(n \geq m)$ random matrix distributed as $\mathcal{C N}_{n, m}\left(\boldsymbol{\Upsilon}, \mathbf{I}_{n} \otimes \boldsymbol{\Sigma}\right)$, where $\boldsymbol{\Sigma}$ is the covariance of the independent complex Gaussian row vectors of $\mathbf{X}$, and $\mathbf{\Upsilon} \in \mathbb{C}^{n \times m}$. Then $\mathbf{W}=\mathbf{X}^{H} \mathbf{X}$ is a complex non-central Wishart matrix that follows the distribution $\mathcal{C} \mathcal{W}_{m}(n, \boldsymbol{\Sigma}, \boldsymbol{\Theta})$ with $\boldsymbol{\Theta}=\boldsymbol{\Sigma}^{-1} \boldsymbol{\Upsilon}^{H} \boldsymbol{\Upsilon}$ the non-centrality parameter.

Concerning the extreme eigenvalues of $\mathbf{W}$, distributional results are available for uncorrelated central $\left(\boldsymbol{\Sigma}=\mathbf{I}_{m}, \boldsymbol{\Upsilon}=\mathbf{0}\right)$, correlated central $(\boldsymbol{\Upsilon}=\mathbf{0})$, and uncorrelated non-central $\left(\boldsymbol{\Sigma}=\mathbf{I}_{m}\right)$ complex Wishart matrices (see, e.g., [29]-[33]). However, tractable results for the correlated non-central case had been unavailable until [8], where the minimum eigenvalue distribution of correlated non-central Wishart matrices has been expressed in terms of $\Phi_{3}$ for some special cases including a square $\mathbf{X}$, i.e., for $m=n$. Specifically, the CDF of $\lambda_{\min }$, the minimum eigenvalue of $\mathbf{W} \sim \mathcal{C} \mathcal{W}_{m}\left(m, \boldsymbol{\Sigma}, \boldsymbol{\Sigma}^{-1} \boldsymbol{\Upsilon}^{H} \boldsymbol{\Upsilon}\right)$, is given when $\Upsilon$ has rank one as [8]

$$
F_{\lambda_{\min }}(\lambda)=1-\exp \left(-\eta-\lambda \operatorname{tr}\left(\boldsymbol{\Sigma}^{-1}\right)\right) \Phi_{3}(m, m, \eta, \lambda \mu)
$$

where $\eta=\operatorname{tr}(\boldsymbol{\Theta})$ and $\mu=\operatorname{tr}\left(\boldsymbol{\Theta} \boldsymbol{\Sigma}^{-1}\right)$.

Analogous expressions to (16), also in terms of $\Phi_{3}$, are found for other special cases such as $2 \times 2$ Wishart matrices with arbitrary degrees of freedom, i.e., $m=2$ with arbitrary $n$, or $3 \times 3$ Wishart matrices with $n=4$ degrees of freedom [8].

Using (16) and Thm. 1, we can express the minimum eigenvalue distribution of correlated non-central Wishart matrices as follows. 
Corollary 3: The CDF of the minimum eigenvalue of $\mathbf{W} \sim \mathcal{C W}_{m}\left(m, \boldsymbol{\Sigma}, \boldsymbol{\Sigma}^{-1} \mathbf{\Upsilon}^{H} \mathbf{\Upsilon}\right)$ with rank-one $\Upsilon$ can be expressed as

$$
\begin{aligned}
F_{\lambda_{\min }}(\lambda)= & 1-\exp \left(-\eta-\lambda \operatorname{tr}\left(\boldsymbol{\Sigma}^{-1}\right)\right) \Gamma(m)\left(\frac{\lambda \mu}{\eta}\right)^{m-1} \\
& \times \sum_{i=0}^{2(m-1)} \mathcal{A}_{i}(m, m ; \lambda \mu) \frac{\eta^{i+1-m}}{(\lambda \mu)^{i}} \exp \left(\eta+\frac{\lambda \mu}{\eta}\right) Q_{2-m+i}\left(\sqrt{2 \eta}, \sqrt{2 \frac{\lambda \mu}{\eta}}\right)
\end{aligned}
$$

where the polynomials $\mathcal{A}_{r}(\cdot, \cdot ; \cdot)$ are as in $(11)$.

Corollary 3 is restricted to the case of a rank-one non-centrality parameter, which however is typically assumed in multiple-input multiple-output (MIMO) communication systems with a direct line-of-sight path between the transmitter and the receiver [34], [35]. Given the complexity of the underlying joint eigenvalue distribution, the CDF in (17) is remarkably simple, involving only a finite sum of generalized Marcum- $Q$ and elementary functions.

The minimum eigenvalue distribution is important in the analysis of MIMO channels [7], [12], [13] where the received signal vector is modeled as

$$
\mathbf{y}=\mathbf{H x}+\mathbf{n}
$$

with $\mathbf{H} \in \mathbb{C}^{N_{\mathrm{r}} \times N_{\mathrm{t}}}$ the channel matrix containing the gains between the $N_{\mathrm{t}}$ transmit and $N_{\mathrm{r}}$ receive antennas, $\mathbf{n} \in \mathbb{C}^{N_{\mathrm{r}} \times 1}$ the noise vector, and $\mathbf{x} \in \mathcal{S}$ the transmitted signal vector with entries drawn from an alphabet $\mathcal{S}$. The minimum eigenvalue of $\mathbf{H}^{H} \mathbf{H}$ determines the minimum distance, $d_{\text {min }}$, between the noiseless received signal vectors and, thereby, the error probability of a MIMO maximum likelihood (ML) receiver. It can be shown that [13]

$$
d_{\min } \geq \sqrt{\lambda_{\min }} d_{0}
$$

where $\lambda_{\min }$ is the minimum eigenvalue of $\mathbf{H}^{H} \mathbf{H}$ and $d_{0}$ is the minimum distance between the elements of $\mathcal{S}$. Altogether, the performance of the MIMO ML receiver is strongly linked to the distribution of $\lambda_{\min }$, which is given in 17 for $\mathbf{H}$ having non-central correlated Gaussian entries; this encompasses both Rayleigh and Rice fading with spatial correlation. Since ML becomes computationally unwieldy as the number of antennas or the transmission alphabet cardinality grows, linear and successive cancellation receivers become attractive. The performance of such receivers also depends on $\lambda_{\min }$ [12], [36]. In fact, the post-receiver SINR of the zero-forcing (ZF), minimum mean square error (MMSE), and Vertical Bell Labs Layered Space-Time (V-BLAST) receivers satisfies [12]

$$
\operatorname{SINR} \geq \frac{E_{\mathrm{s}}}{N_{\mathrm{t}} \sigma^{2}} \lambda_{\min }
$$


where $E_{\mathrm{s}}$ is the energy per symbol, i.e., $\mathrm{E}\left[\mathbf{x x}^{H}\right]=\left(E_{\mathrm{s}} / N_{\mathrm{t}}\right) \mathbf{I}_{N_{\mathrm{t}}}$, and $\sigma^{2}$ is the noise variance.

Combining Corollary 3 with (20), the outage probability of MIMO receivers can be analyzed in fairly broad generality. In addition, Corollary 3 has further applications, e.g., in the design and analysis of adaptive MIMO multiplexing-diversity switching [7] or, in the context of econometrics, in characterizing the weak instrument asymptotic distribution of the Cragg-Donald statistic [37].

\section{ACKNOWLEDGEMENTS}

The work of D. Morales-Jimenez and A. Lozano has been supported by the Spanish Government under projects TEC2012-34642, CSD2008-00010 (Consolider-Ingenio) and by the Catalan Government (SGR2009\#70). The work of F. J. Lopez-Martinez is supported by the University of Malaga and by the European Union under Marie-Curie COFUND U-mobility program (ref. 246550). The work of E. Martos-Naya and J. F. Paris has been supported by the Spanish Government-FEDER under projects TEC2010-18451 and TEC2011-25473.

\section{APPENDIX A}

\section{PROOF OF LEMMA 1}

The generalized Marcum- $Q$ function can be obtained as the contour integral in the complex plane [38]

$$
Q_{m}(a, b)=\exp \left(-\frac{a^{2}+b^{2}}{2}\right) \underbrace{\oint_{\Gamma_{0}} \frac{1}{p^{m}(1-p)} \exp \left(\frac{1}{2}\left(\frac{a^{2}}{p}+b^{2} p\right)\right) d p}_{\mathcal{I}_{m}(a, b)}
$$

where $\oint_{\Gamma_{0}} \triangleq \frac{1}{2 \pi j} \int_{\Gamma_{0}}$ and $\Gamma_{0}$ is any closed contour enclosing the singularity at $p=0$ (in a counterclockwise direction) and no other singularities of the integrand (cf. Fig. 11. For convenience, we express the integral $\mathcal{I}_{m}(a, b)$ in 21 as

$$
\mathcal{I}_{m}(a, b)=\oint_{\Gamma_{0}} F(p) \exp \left(\frac{b^{2} p}{2}\right) d p
$$

with

$$
F(p)=\frac{1}{1-p} p^{-m} \exp \left(\frac{a^{2}}{2 p}\right)
$$

Let us consider the contour $C$ depicted in Fig. 1, where $c$ is chosen to the right of all the singularities of $F(p)$. Letting $R \rightarrow \infty$, the contour integral along $C$ equals

$$
\oint_{C} F(p) \exp \left(\frac{b^{2} p}{2}\right) d p=\frac{1}{2 \pi j} \int_{c-j \infty}^{c+j \infty} F(p) \exp \left(\frac{b^{2} p}{2}\right) d p+\oint_{C_{\beta}} F(p) \exp \left(\frac{b^{2} p}{2}\right) d p .
$$




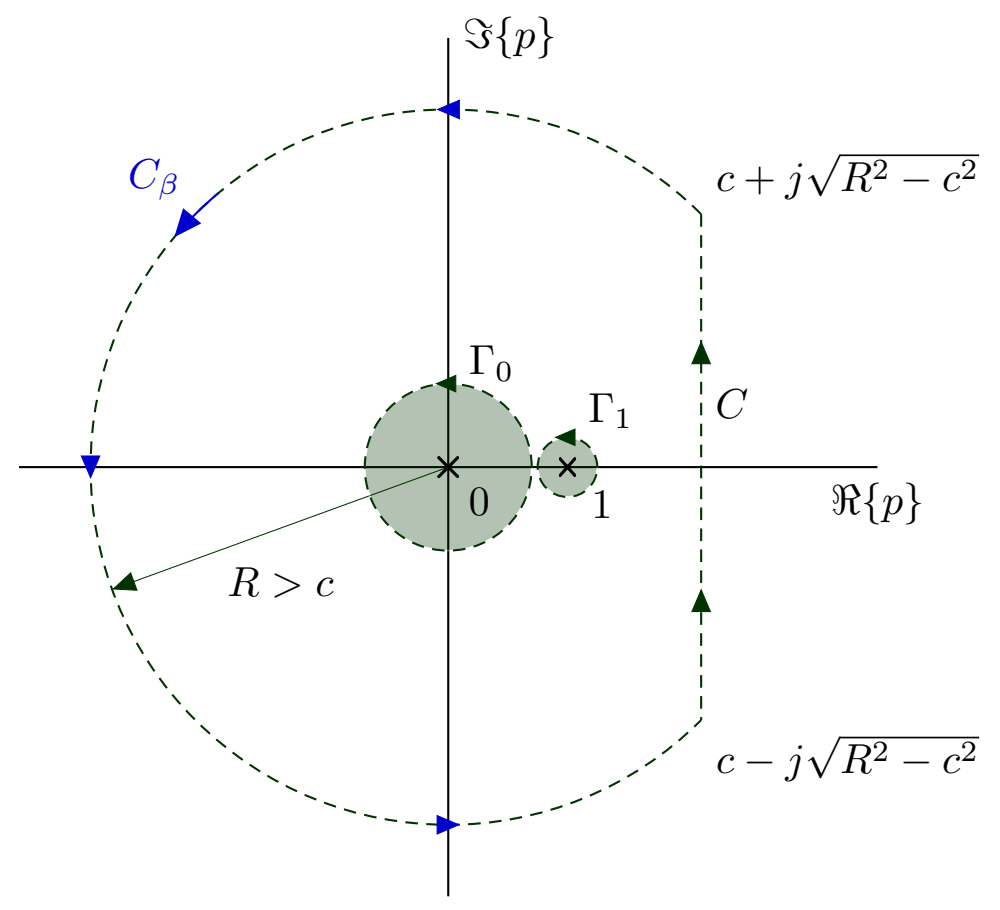

Fig. 1. Contour integration for integral $\mathcal{I}_{m}$.

Alternatively, we can apply the Cauchy-Goursat theorem to obtain

$$
\oint_{C} F(p) \exp \left(\frac{b^{2} p}{2}\right) d p=\oint_{\Gamma_{0}} F(p) \exp \left(\frac{b^{2} p}{2}\right) d p+\oint_{\Gamma_{1}} F(p) \exp \left(\frac{b^{2} p}{2}\right) d p
$$

where $\Gamma_{0}$ and $\Gamma_{1}$ are closed contours enclosing the singularities at $p=0$ and $p=1$, respectively. Combining (24) and 25),

$$
\frac{1}{2 \pi j} \int_{c-j \infty}^{c+j \infty} F(p) \exp \left(\frac{b^{2} p}{2}\right) d p+\oint_{C_{\beta}} F(p) \exp \left(\frac{b^{2} p}{2}\right) d p=\oint_{\Gamma_{0}+\Gamma_{1}} F(p) \exp \left(\frac{b^{2} p}{2}\right) d p .
$$

The first integral in 26 is related to the inverse Laplace transform of $F(p)$ as

$$
\frac{1}{2 \pi j} \int_{c-j \infty}^{c+j \infty} F(p) \exp \left(\frac{b^{2} p}{2}\right) d p=\left.\mathcal{L}^{-1}\{F(p) ; t\}\right|_{t=\frac{b^{2}}{2}} .
$$

The integral along $C_{\beta}$ can be shown to be zero as follows. The modulus of $F(p)$ in $C_{\beta}$ is

$$
|F(p)|_{p=\operatorname{Re}^{j \theta}}=\frac{1}{|1-p|} \frac{1}{|p|^{m}}\left|e^{\delta / p}\right|
$$

with $\delta=a^{2} / 2$. Then, we can use the inequalities

$$
\begin{aligned}
\frac{1}{|1-p|} & \leq \frac{1}{|1-| p||} \underset{R>2}{\leq} \frac{2}{R} \\
\left|e^{\delta / p}\right| & =\left|e^{\operatorname{Re}(\delta / p)}\right| \leq e^{|\delta / R|} \underset{R>R_{0}}{\leq} e^{\left|\delta / R_{0}\right|}
\end{aligned}
$$


with arbitrary (finite) $R_{0}$, to write

$$
|F(p)| \underset{R>R_{0}}{\leq} \underbrace{2 e^{\left|\delta / R_{0}\right|}}_{K} \underbrace{R^{-(m+1)}}_{R^{-\ell}} .
$$

Thus, $|F(p)|_{p=R e^{j \theta}} \leq K R^{-\ell}$ for some $\ell>0$ on $C_{\beta}$ and, according to Jordan's lemma, the integral $\oint_{C_{\beta}}$ equals 0 as $R \rightarrow \infty$. Plugging (22) and (27) into (26) and applying the residue theorem, we arrive at

$$
\mathcal{I}_{m}(a, b)=\mathcal{L}^{-1}\{F(p) ; t\}_{t=\frac{b^{2}}{2}}-\operatorname{Res}\left\{F(p) \exp \left(\frac{b^{2} p}{2}\right)\right\}_{p=1}
$$

where $\operatorname{Res}\{\cdot\}_{p=\xi}$ denotes the residue at $p=\xi$. Then, we calculate the residue and use 8 to solve the inverse Laplace transform, which yields

$$
\mathcal{I}_{m}(a, b)=-\left(\frac{b^{2}}{2}\right)^{m} \tilde{\Phi}_{3}\left(1, m+1 ; \frac{b^{2}}{2}, \frac{a^{2} b^{2}}{4}\right)+\exp \left(\frac{a^{2}+b^{2}}{2}\right) .
$$

Further substituting (33) in 21) gives the alternative form for the generalized Marcum- $Q$ function,

$$
Q_{m}(a, b)=1-\left(\frac{b^{2}}{2}\right)^{m} \exp \left(-\frac{a^{2}+b^{2}}{2}\right) \tilde{\Phi}_{3}\left(1, m+1 ; \frac{b^{2}}{2}, \frac{a^{2} b^{2}}{4}\right)
$$

which is valid for any $m \in \mathbb{R}$.

Finally, (9) is obtained by combining (34) and (2), completing the proof.

\section{APPENDIX B}

\section{ProOF OF LEMMA 2}

The recursive relationship in $(10)$ is derived by using $(8)$ and the frequency differentiation property of the Laplace transform,

$$
\mathcal{L}\{t \cdot f(t)\}=-\frac{d F(s)}{d s} .
$$

Taking the first derivative of (8), in light of (35),

$$
\begin{aligned}
& t^{c} \tilde{\Phi}_{3}(b, c ; x t, y t)= \\
& \quad \mathcal{L}^{-1}\left\{e^{y / s}\left(b x s^{-(c+2)}\left(1-\frac{x}{s}\right)^{-(b+1)}+c s^{-(c+1)}\left(1-\frac{x}{s}\right)^{-b}+y s^{-(c+2)}\left(1-\frac{x}{s}\right)^{-b}\right)\right\}
\end{aligned}
$$

where $\mathcal{L}^{-1}\{\cdot\}$ stands for the inverse Laplace transform. Then, with the help of 8 , we can identify the right-hand side of 36 as a sum of $\tilde{\Phi}_{3}$ functions, which allows us to write (after some algebra)

$$
\begin{aligned}
& \tilde{\Phi}_{3}(b, c ; x t, y t)= \\
& \quad \frac{1}{(b-1) x t}\left(\tilde{\Phi}_{3}(b-1, c-2 ; x t, y t)-(c-2) \tilde{\Phi}_{3}(b-1, c-1 ; x t, y t)-y t \tilde{\Phi}_{3}(b-1, c ; x t, y t)\right)
\end{aligned}
$$


which shows that $\tilde{\Phi}_{3}$ can be recursively expressed via lower values of its first argument. Thus, 37 can be recursively applied to yield

$$
\tilde{\Phi}_{3}(b, c ; x t, y t)=\sum_{i=0}^{2(b-1)} \alpha_{i} \tilde{\Phi}_{3}(1, c-i ; x t, y t)
$$

where $\alpha_{i}$ are certain coefficients associated to $\tilde{\Phi}_{3}(1, c-i ; x t, y t)$ that can be obtained by working out the recursion (37). An explicit formula for $\alpha_{i}$ can be inferred from the first coefficients $\alpha_{i}, i=0,1,2, \ldots$, leading to

$$
\alpha_{i}=\frac{(-1)^{b-1}}{\Gamma(b)} \frac{(y t)^{b-1-i}}{(x t)^{b-1}} \sum_{k=0}^{\lfloor i / 2\rfloor} \frac{(-1)^{k}(b-i+k)_{i-k}(c-i-1+k)_{i-2 k}}{(i-2 k) ! k !}(y t)^{k} .
$$

Finally, (10)-(11) follow from (38)-(39) after setting $x t=w, y t=z$, and defining the polynomial $\mathcal{A}_{i}(b, c ; z)=\left(w^{b-1} / z^{b-1-i}\right) \alpha_{i}$.

\section{REFERENCES}

[1] G. Di Blasio and A. Neri, "Modified Q-functions and their use in detection analysis," IEEE Trans. Inf. Theory, vol. 37, no. 4, pp. 1123-1142, Jul. 1991.

[2] A. Baricz and Y. Sun, "New bounds for the generalized Marcum Q-function," IEEE Trans. Inf. Theory, vol. 55, no. 7, pp. 3091-3100, Jul. 2009.

[3] G. Corazza and G. Ferrari, "New bounds for the Marcum Q-function," IEEE Trans. Inf. Theory, vol. 48, no. 11, pp. 3003-3008, Nov. 2002.

[4] V. M. Kapinas, S. K. Mihos, and G. K. Karagiannidis, "On the monotonicity of the generalized Marcum and Nuttall $Q$ -functions," IEEE Trans. Inf. Theory, vol. 55, no. 8, pp. 3701-3710, Aug. 2009.

[5] A. B. Yin Sun and S. Zhou, "On the monotonicity, log-concavity, and tight bounds of the generalized Marcum and Nuttall Q-functions," IEEE Trans. Inf. Theory, vol. 56, no. 3, pp. 1166-1186, Mar. 2010.

[6] G. Alfano, A. De Maio, and A. Tulino, "A theoretical framework for LMS MIMO communication systems performance analysis," IEEE Trans. Inf. Theory, vol. 56, no. 11, pp. 5614-5630, Nov. 2010.

[7] J. Heath, R.W. and D. Love, "Multimode antenna selection for spatial multiplexing systems with linear receivers," IEEE Trans. Signal Process., vol. 53, no. 8, pp. 3042-3056, Aug. 2005.

[8] P. Dharmawansa and M. R. McKay, "Extreme eigenvalue distributions of some complex correlated non-central Wishart and gamma-Wishart random matrices," J. Mult. Anal., vol. 102, no. 4, pp. 847-868, 2011.

[9] D. Morales-Jimenez, J. Paris, and A. Lozano, “Outage probability analysis for MRC in $\eta-\mu$ fading channels with co-channel interference," IEEE Commun. Lett., vol. 16, no. 5, pp. 674-677, May 2012.

[10] F. Chatelain, J. Y. Tourneret, J. Inglada, and A. Ferrari, "Bivariate gamma distributions for image registration and change detection,” IEEE Trans. Image Process., vol. 16, no. 7, pp. 1796-1806, Jul. 2007.

[11] F. J. Lopez-Martinez, D. Morales-Jimenez, E. Martos-Naya, and J. F. Paris, "On the bivariate Nakagami-m cumulative distribution function: closed-form expression and applications," IEEE Trans. Commun., to appear 2013.

[12] R. Narasimhan, "Spatial multiplexing with transmit antenna and constellation selection for correlated MIMO fading channels," IEEE Trans. Signal Process., vol. 51, no. 11, pp. 2829-2838, Nov. 2003. 
[13] G. Burel, "Statistical analysis of the smallest singular value in MIMO transmission systems," in In Proc. of the WSEAS Int. Conf. on Signal, Speech and Image Processing (ICOSSIP), 2002.

[14] M. K. Simon and M.-S. Alouini, Digital Communications over Fading Channels, 2nd ed. John Wiley, 2005.

[15] C. Tan and N. Beaulieu, "Infinite series representations of the bivariate Rayleigh and Nakagami- $m$ distributions," IEEE Trans. Commun., vol. 45, no. 10, pp. 1159-1161, Oct. 1997.

[16] J. Reig, L. Rubio, and N. Cardona, "Bivariate Nakagami-m distribution with arbitrary fading parameters," Electron. Lett., vol. 38, no. 25 , pp. 1715-1717, Dec. 2002.

[17] R. de Souza and M. Yacoub, "Bivariate Nakagami- $m$ distribution with arbitrary correlation and fading parameters," IEEE Trans. Wireless Commun., vol. 7, no. 12, pp. 5227-5232, Dec. 2008.

[18] N. Beaulieu and K. Hemachandra, "Novel simple representations for Gaussian class multivariate distributions with generalized correlation,” IEEE Trans. Inf. Theory, vol. 57, no. 12, pp. 8072-8083, Dec. 2011.

[19] A. A. Abu-Dayya and N. C. Beaulieu, "Analysis of switched diversity systems on generalized-fading channels," IEEE Trans. Commun., vol. 42, no. 11, pp. 2959-2966, Nov. 1994.

[20] W. C. Jakes, Ed., Microwave Mobile Communications. New York: IEEE Press, 1974.

[21] M. Zorzi, R. Rao, and L. Milstein, "Error statistics in data transmission over fading channels," IEEE Trans. Commun., vol. 46, no. 11, pp. 1468-1477, Nov. 1998.

[22] H.-S. Wang and P.-C. Chang, "On verifying the first-order Markovian assumption for a Rayleigh fading channel model," IEEE Trans. Veh. Technol., vol. 45, no. 2, pp. 353-357, May 1996.

[23] I. S. Gradshteyn and I. M. Ryzhik, Table of Integrals, Series, and Products, 6th ed. San Diego: Academic Press, 2000.

[24] A. Erdelyi, High Order Transcendental Functions. McGraw-Hill, 1953, vol. 1.

[25] J. Marcum, "Table of $Q$ functions," Rand Corporation, Santa Monica, CA, U.S. Air Force Project RAND Research Memorandum M-339, ASTIA Document AD 1165451, Jan. 1950.

[26] A. Nuttall, "Some integrals involving the $Q_{M}$ function," IEEE Trans. Inf. Theory, vol. 21, no. 1, pp. 95-96, Jan. 1975.

[27] C. O'Driscoll and C. Murphy, "A simplified expression for the probability of error for binary multichannel communications," IEEE Trans. Commun., vol. 57, no. 1, pp. 32-35, Jan. 2009.

[28] M. Schwartz, W. R. Bennett, and S. Stein, Communication systems and techniques. New York: IEEE Press, 1966.

[29] Y. Chen and S. Manning, "Some eigenvalue distribution functions of the Laguerre ensemble," Journal of Physics A: Mathematical and General, vol. 29, no. 23, p. 7561, 1999.

[30] M. Chiani, M. Win, and A. Zanella, "On the capacity of spatially correlated MIMO Rayleigh-fading channels," IEEE Trans. Inf. Theory, vol. 49, no. 10, pp. 2363-2371, Oct. 2003.

[31] P. Forrester, "Eigenvalue distributions for some correlated complex sample covariance matrices," Journal of Physics A: Mathematical and Theoretical, vol. 40, no. 36, pp. 11093-11 103, 2007.

[32] A. Maaref and S. Aissa, "Joint and marginal eigenvalue distributions of (non)central complex Wishart matrices and PDFbased approach for characterizing the capacity statistics of MIMO Ricean and Rayleigh fading channels," IEEE Trans. Wireless Commun., vol. 6, no. 10, pp. 3607-3619, Oct. 2007.

[33] A. Zanella, M. Chiani, and M. Win, "On the marginal distribution of the eigenvalues of Wishart matrices," IEEE Trans. Commun., vol. 57, no. 4, pp. 1050-1060, Apr 2009.

[34] S. Jayaweera and H. Poor, "On the capacity of multiple-antenna systems in Rician fading," IEEE Trans. Wireless Commun., vol. 4, no. 3, pp. 1102-1111, May 2005. 
[35] Y. Zhu, P.-Y. Kam, and Y. Xin, "On the mutual information distribution of MIMO Rician fading channels," IEEE Trans. Commun., vol. 57, no. 5, pp. 1453-1462, May 2009.

[36] D. Gore, R. Heath, and A. Paulraj, "Statistical antenna selection for spatial multiplexing systems," in IEEE Int. Conf. Commun. (ICC 2002), 2002, pp. 450-454.

[37] J. H. Stock and M. Yogo, "Testing for weak instruments in linear IV regression,” National Bureau of Economic Research, Working Paper 284, Nov. 2002.

[38] J. Proakis, Digital Communications, 4th ed. McGraw-Hill, Aug. 2000. 